\title{
Preface of the "Symposium on Dynamical Systems Applied to Robotics"
}

\author{
Cristina P. Santos ${ }^{\mathrm{a}}$ and Carla M.A. Pinto ${ }^{\mathrm{b}}$ \\ ${ }^{a}$ Universidade do Minho Dept. Electrónica Industrial \\ Campus de Azurém 4800-058 Guimarães \\ Portugal \\ ${ }^{b}$ Instituto Superior de Engenharia do Porto \\ and Centro de Matemática da Universidade do Porto \\ Rua Dr António Bernardino de Almeida, 431, \\ 4200-072 Porto, Portugal
}

Robotics has been evolving in last years, considering the different fields that can be tackled. This is an important and diversified domain, which is normally solved using bio-inspired or model-based approaches.

In this symposia, we focus on the use of the dynamical systems theory to address any related problems in the robotics domain. Dynamical systems exhibit some properties which makes them well suited to real-time robotic applications, such as movement generation for legged; modular and wheeled platforms; balance control; grasping; reaching, etc.

The organizers aim to provide a venue where researchers, scientists, engineers and practitioners throughout the world can present and discuss the latest achievements, future challenges and exciting new dynamical systems models that will enable to improve the achievements of future robots.

\section{Cristina Santos}

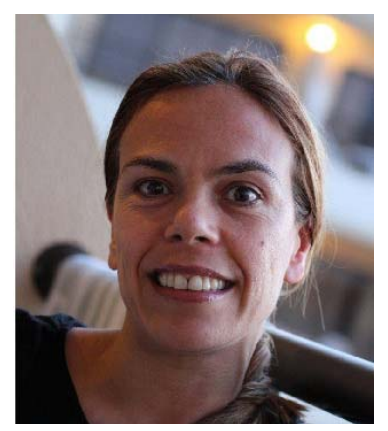

Doctor Cristina received the B.S. degree in Industrial Electronics, the M.Sc degree in Robotics, and the Ph.D. degree in Robotics in the field of Nonlinear dynamics, all from University of Minho, Guimaraes, Portugal, in 1994, 1998 and 2003 respectively. The PhD was also in collaboration with the CNRS-CNRC Marseille, France.

She is working since 1996 as an Auxiliar Professor at the University of Minho, Industrial Electronics Department, Portugal. Her research focus on the extension of the use of the dynamical systems theory to the achievement of more complex behavior for robots: generate locomotion for multi-dof robots; achieve cooperativity among multi-robots and learning. Recently her research interests focus on methods to characterize human motion, and designing robots and robot controllers for rehabilitation of patients suffering from motor problems.

She has authored/co-authored more than 40 research papers, published in international journals or presented at international conferences.

\section{Carla Pinto}

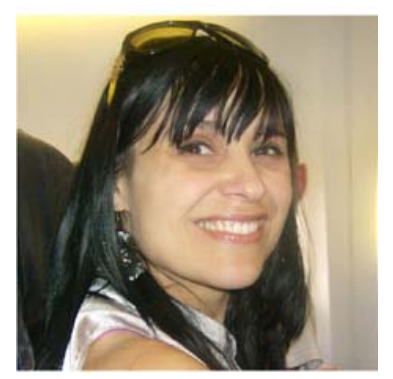

Carla Pinto is an Assistant Professor at Instituto Superior de Engenharia do Porto, Portugal, since December 1997, and does research at Centro de Matemática da Universidade do Porto. She is a Doctor in Mathematics (since 2004). Her current research fields involve the study of nonlinear dynamical systems, considering fractional and integer derivatives, using bifurcation theory and symmetry techniques, and the study of online generation of trajectories in robots using a dynamical systems approach. Some previous work involved the study of coupled dynamical systems as models of biological systems, namely animal locomotion, neurons.

She has authored/co-authored a large number of research papers, published in international journals or presented at international conferences. 